$(4)^{*}$

$$
\begin{aligned}
& p=\left(\frac{a+2 b+5 c+10 d}{6}\right)^{2}+2\left(\frac{a-b+5 c-5 d}{6}\right)^{2} \\
& +5\left(\frac{a+2 b-c-2 d}{6}\right)^{2}+10\left(\frac{a-b-c+d}{6}\right)^{2}
\end{aligned}
$$

In view of (2) and (3), the numerators in (4) are all even. Then, unless exactly three of $a, b, c, d$ are divisible by 3 , we can choose signs for $a, b, c, d$ so that

$$
a-b-c+d \equiv 0(\bmod 3) \text {. }
$$

Then all the other numerators in (4) are divisible by 3 .

In the exceptional case either $a$ and $b$ or $c$ and $d$ are divisible by 3 . But the identity

$$
9\left(A^{2}+2 B^{2}\right)=(A \pm 4 B)^{2}+2(2 A \mp B)^{2}
$$

(repeated if necessary) shows that any multiple of 3 of the form $x^{2}+2 y^{2}$ can be expressed in that form with $x, y$ prime to 3 . Then (5) can be verified as above, and $q=1$. We have now proved the following theorem:

THEOREM 4. Every positive integer is representable in the form

$$
a^{2}+2 b^{2}+5 c^{2}+10 d^{2} \text {. }
$$

UNIVERSITY OF ILLINOIS

\title{
A MOMENT-GENERATING FUNCTION WHICH IS USEFUL IN SOLVING CERTAIN MATCHING PROBLEMS $\dagger$
}

EDWIN G. OLDS

1. Introduction. In a book published several years ago, Fry $\ddagger$ devoted considerable attention to various aspects of a problem which he called, "the psychic research problem." His introductory problem is the following: "A spiritualistic medium claims to be able to tell the

* Formula (4) and the rest of the proof of this theorem were suggested by Gordon Pall.

$\dagger$ Presented to the Society and The Institute of Mathematical Statistics, December 30, 1937.

$\ddagger$ T. C. Fry, Probability and Its Engineering Uses, Van Nostrand, New York, 1928, pp. 41-77. 
color of a playing card without seeing it. In order to test her claims an experiment is conducted with four red and four black cards. These cards are thoroughly shuffled and placed face down on the table. The medium is told that there are four red and four black cards, but presumably knows nothing as to their arrangement. The experimenter picks up a card and, without either looking at it himself or showing it to the medium, asks its color. If she answers 'red,' he places it at one side of the table. If she answers 'black,' he places it on the other side of the table. This process is repeated until all cards are exhausted. If the medium does not have the ability which she claims to possess, what is the chance that there will be just one black card in the pile that should be red?"

Fry solves this problem by considering the number of chance orders of red and black which would match the order in which the medium calls the cards to the extent of producing exactly three red pairs. $\mathrm{He}$ also proposes and solves various other problems of this general type. A problem like Fry's, but concerned with tea-tasting, is thoroughly discussed by Fisher.*

Chapman $\dagger$ in 1931, contributed the solutions of several problems related to the expected number of correct matchings if a deck of $t$ different cards is placed in correspondence with a given target deck of the same kind. One of his results is the function

$$
p(s)=\frac{1}{s !}\left[\frac{1}{0 !}-\frac{1}{1 !}+\frac{1}{2 !}-\cdots+(-1)^{t-s} \frac{1}{(t-s) !}\right],
$$

which gives the probability of $s$ correct matchings.

A more general problem has been considered by Greenwood. $\ddagger \mathrm{He}$ considers matching a target deck containing $t$ sets of identical cards, with $s_{i}$ cards in the $i$ th set, $(i=1,2, \cdots, t)$. For the case where the number of cards is the same for each set, he obtains the mean and variance of the distribution of the numbers of correct matchings as $s$ and $s^{2}(t-1) /(s t-1)$, respectively. The purpose of the present paper is to develop a moment-generating function and derive the first four moments for the theoretical distribution of numbers of correct match-

${ }^{*}$ R. A. Fisher, The Design of Experiments, Oliver and Boyd, Edinburgh, 1935, chap. 2 .

$\dagger \mathrm{D}$. W. Chapman, The statistics of the method of correct matchings, American Journal of Psychology, vol. 46 (1934), pp. 287-298.

$\ddagger \mathrm{J}$. A. Greenwood, Variance of a general matching problem, Annals of Mathematical Statistics, vol. 9 (1938), pp. 56-59. See also M. S. Bartlett, Properties of sufficiency and statistical tests, Proceedings of the Royal Society, (A), vol. 160, pp. 268-282 (note especially the discussion of the $l \times m$ contingency table, pp. 278-280). 
ings for this case where the number of cards is the same for each set.*

2. Discussion of Fry's problem. Before taking up the general case of $t$ sets of $s$ cards each, let us consider a solution of Fry's problem, which we quoted above.

Suppose the cards are tagged $R_{1}, R_{2}, R_{3}, R_{4}, B_{1}, B_{2}, B_{3}, B_{4}$. Let us consider the order in which the medium calls the cards as constituting the order in which a target deck is laid out. When the deck used for matching is laid out in correspondence to the target deck, any of the cards of the matching deck may fall below any one of the cards of the target deck. This is illustrated in the array below, where the columns correspond to the cards of the target deck and the entries in

\begin{tabular}{|c|c|c|c|c|c|c|c|}
\hline$R_{1}$ & $R_{2}$ & $R_{3}$ & $R_{4}$ & $B_{1}$ & $B_{2}$ & $B_{3}$ & $B_{4}$ \\
\hline$R_{1}$ & $\overline{R_{1}}$ & $R_{1}$ & $R_{1}$ & $R_{1}$ & $R_{1}$ & $R_{1}$ & $R_{1}$ \\
\hline$R_{2}$ & $R_{2}$ & $R_{2}$ & $R_{2}$ & $R_{2}$ & $R_{2}$ & $R_{2}$ & $R_{2}$ \\
\hline$R_{3}$ & $R_{3}$ & $R_{3}$ & $R_{3}$ & $R_{3}$ & $R_{3}$ & $R_{3}$ & $R_{3}$ \\
\hline$R_{4}$ & $R_{4}$ & $R_{4}$ & $R_{4}$ & $R_{4}$ & $R_{4}$ & $R_{4}$ & $R_{4}$ \\
\hline$B_{1}$ & $B_{1}$ & $B_{1}$ & $B_{1}$ & $B_{1}$ & $B_{1}$ & $B_{1}$ & $B_{1}$ \\
\hline$B_{2}$ & $B_{2}$ & $B_{2}$ & $B_{2}$ & $B_{2}$ & $B_{2}$ & $B_{2}$ & $B_{2}$ \\
\hline$B_{3}$ & $B_{3}$ & $B_{3}$ & $B_{3}$ & $B_{3}$ & $B_{3}$ & $B_{3}$ & $B_{3}$ \\
\hline$B_{4}$ & $B_{4}$ & $B_{4}$ & $B_{4}$ & $B_{4}$ & $\overline{B_{4}}$ & $B_{4}$ & $B_{4}$ \\
\hline
\end{tabular}

Array I

\begin{tabular}{|c|c|c|c|c|c|c|c|}
\hline$R_{1}$ & $R_{2}$ & $R_{3}$ & $R_{4}$ & $B_{1}$ & $B_{2}$ & $B_{3}$ & $B_{4}$ \\
\hline$x$ & $x$ & $x$ & $x$ & 1 & 1 & 1 & 1 \\
\hline$x$ & $x$ & $x$ & $x$ & 1 & 1 & 1 & 1 \\
\hline$x$ & $x$ & $x$ & $x$ & 1 & 1 & 1 & 1 \\
\hline$x$ & $x$ & $x$ & $x$ & 1 & 1 & 1 & 1 \\
\hline 1 & 1 & 1 & 1 & $x$ & $x$ & $x$ & $x$ \\
\hline 1 & 1 & 1 & 1 & $x$ & $x$ & $x$ & $x$ \\
\hline 1 & 1 & 1 & 1 & $x$ & $x$ & $x$ & $x$ \\
\hline 1 & 1 & 1 & 1 & $x$ & $x$ & $x$ & $x$ \\
\hline
\end{tabular}

Array II

the columns correspond to the cards of the matching deck. Now, whenever $R_{1}$ appears in a column labeled $R_{1}, R_{2}, R_{3}$, or $R_{4}$, this represents a matched pair; and likewise other reds in the red columns and the blacks in the black columns represent matched pairs. In every such case let us replace the symbol by $x$, and in all other cases by 1 . In this way we form the second array.

Now, if we make all possible choices of one element from each column of the first array, with no two from any row, we have all of the 8 ! orders in which the cards of the matching deck may appear. Let us suppose that one of these is $R_{1} R_{2} B_{1} B_{3} R_{3} R_{4} B_{2} B_{4}$. This corresponds to $x, x, 1,1,1,1, x, x$ in the second array, or, if we drop the

* T. E. Sterne, The solution of a problem in probability, Science, vol. 86, pp. 500501 , has calculated the first four moments for the case $s=5, t=5$; also note numerical results given by E. V. Huntington, Exact probabilities in certain card-matching problems, Science, vol. 86, pp. 499-500; and results given by C. E. Kellogg, New evidence(?) for extra-sensory perception, Scientific Monthly, vol. 45 (1937), pp. 331-341. 
commas and multiply, it corresponds to $x^{4}$, where the exponent indicates that we have exactly four matched pairs. Forming every possible product from the second array, using only one element from each column and no two from any row, we have 8 ! terms of various degrees. Collecting like powers we have

$$
\phi(x)=(4 !)^{2} x^{8}+16(4 !)^{2} x^{6}+36(4 !)^{2} x^{4}+16(4 !)^{2} x^{2}+(4 !)^{2},
$$

which means that, of all the possible arrangements, $(4 !)^{2}$ produce eight matched pairs, $16(4 !)^{2}$ produce six matched pairs, for example. If we divide the coefficients by 8 ! we have the probabilities. Thus Fry's problem, which requires the probability for six matched pairs, has the answer $16(4 !)^{2} / 8$ ! or $8 / 35$.

If we denote by $f(r)$ the frequency of $r$ matched pairs, we may write the above polynomial in the form

$$
\phi(x)=\sum_{r=0}^{8} f(r) x^{r} .
$$

Then, obviously, $\phi(x) / 8$ ! is a generating function for factorial moments. Denoting the factorial moments by $K$, where

$$
K_{i}=\frac{\sum_{r=0}^{8} r^{(i)} f(r)}{8 !}, \quad \text { if } \quad r^{(i)}=r(r-1) \cdots(r-i+1),
$$

we have

$$
K_{0}=\phi(1) / 8 !, K_{1}=\phi^{\prime}(1) / 8 !, \cdots, K_{i}=\phi^{i}(1) / 8 ! .
$$

The form of the function $\phi(x)$ needs detailed consideration. This polynomial was obtained from the array of $x$ 's and 1's by adding all the terms formed by choosing one element from each column but no two from any row. Such an array has been called a permanent, ${ }^{*}$ and the symbol +||$+$ has been used for it. The properties of the permanent which will be useful in this paper are given in the next section.

3. The permanent. It is obvious that the permanent has properties analogous to those properties of the determinant which do not depend on the fact that the determinant vanishes when two rows (or two columns) are identical. For example, the permanent can be expanded in terms of minor permanents. Of particular importance is the fact that the derivative of a permanent in one variable is equal to the sum

* T. Muir, revised by W. H. Metzler, A Treatise on the Theory of Determinants, Muir, Albany, 1930, p. 19. 
of the permanents formed by replacing, one column at a time, the elements of each column by their derivatives.

4. Application of the permanent. As noted above, we may identify $\phi(x)$ as a permanent, namely

$$
\phi(x)=\left|\begin{array}{llllllll}
x & x & x & x & 1 & 1 & 1 & 1 \\
x & x & x & x & 1 & 1 & 1 & 1 \\
x & x & x & x & 1 & 1 & 1 & 1 \\
x & x & x & x & 1 & 1 & 1 & 1 \\
1 & 1 & 1 & 1 & x & x & x & x \\
1 & 1 & 1 & 1 & x & x & x & x \\
1 & 1 & 1 & 1 & x & x & x & x \\
1 & 1 & 1 & 1 & x & x & x & x
\end{array}\right|+
$$

Obviously, $\phi(1)=8$ !. The first term of $\phi^{\prime}(x)$ is

$$
+\left|\begin{array}{llllllll}
1 & x & x & x & 1 & 1 & 1 & 1 \\
1 & x & x & x & 1 & 1 & 1 & 1 \\
1 & x & x & x & 1 & 1 & 1 & 1 \\
1 & x & x & x & 1 & 1 & 1 & 1 \\
0 & 1 & 1 & 1 & x & x & x & x \\
0 & 1 & 1 & 1 & x & x & x & x \\
0 & 1 & 1 & 1 & x & x & x & x \\
0 & 1 & 1 & 1 & x & x & x & x
\end{array}\right|+4 \cdot\left|\begin{array}{lllllll}
x & x & x & 1 & 1 & 1 & 1 \\
x & x & x & 1 & 1 & 1 & 1 \\
x & x & x & 1 & 1 & 1 & 1 \\
1 & 1 & 1 & x & x & x & x \\
1 & 1 & 1 & x & x & x & x \\
1 & 1 & 1 & x & x & x & x \\
1 & 1 & 1 & x & x & x & x
\end{array}\right|,
$$

and it is easy to see that each of the other seven terms is equivalent to this one. Therefore

$$
\phi^{\prime}(x)=4 \cdot 8 \cdot\left|\begin{array}{lllllll}
x & x & x & 1 & 1 & 1 & 1 \\
x & x & x & 1 & 1 & 1 & 1 \\
x & x & x & 1 & 1 & 1 & 1 \\
1 & 1 & 1 & x & x & x & x \\
1 & 1 & 1 & x & x & x & x \\
1 & 1 & 1 & x & x & x & x \\
1 & 1 & 1 & x & x & x & x
\end{array}\right| \text {, and } \phi^{\prime}(1)=4 \cdot 8 \cdot 7 !=4 \cdot 8 ! \text {. }
$$

In the permanent above, one of the blocks of $x$ 's has one less column and one less row than before. It is convenient, then, to denote 
this permanent by [1]. Using the same system, we will denote by [21] the permanent, where one block of $x$ 's is reduced by two rows and two columns and the other by one row and one column.

It can be readily verified that $\phi^{\prime \prime}(x)=4 \cdot 8(3 \cdot 3[2]+4 \cdot 4[11])$ and $\phi^{\prime \prime}(1)=4 \cdot 8\left(3^{2}+4^{2}\right) \cdot 6 !=\left(4 \cdot 5^{2} \cdot 8 !\right) / 7$. The succeeding derivatives may be written in similar fashion.

Using these results, we may compute the factorial moments and, from them, the moments about the mean.

5. The solution of the general problem. For the general problem we have $t$ groups (instead of 2) of $s$ like cards each (instead of 4). The general method of obtaining the moment-generating function, and from it the moments, is the same as was used for the special case, except that the work becomes somewhat more intricate and arduous. In the general case the function $\phi(x)$ is a permanent of $n$ rows and $n$ columns, $(s t=n)$, and has $t$ blocks of $s x$ 's each. Then

$$
\begin{aligned}
\phi(x) & =[0], \\
\phi^{\prime}(x) & =s^{2} t[1], \\
\phi^{\prime \prime}(x) & =\left(s^{(2)}\right)^{2} t[2]+s^{4} t^{(2)}\left[1^{2}\right], \\
\phi^{\prime \prime \prime}(x) & =\left(s^{(3)}\right)^{2} t[3]+3\left(s^{(2)} s\right)^{2} t^{(2)}[21]+s^{6} t^{(3)}\left[1^{3}\right], \\
\phi^{\mathrm{iv}}(x)= & \left(s^{(4)}\right)^{2} t[4]+4\left(s^{(3)} s\right)^{2} t^{(2)}[31]+3\left(s^{(2)}\right)^{4} t^{(2)}\left[2^{2}\right] \\
& +6\left(s^{(2)} s^{2}\right)^{2} t^{(3)}\left[21^{2}\right]+\left(s^{4}\right)^{2} t^{(4)}\left[1^{4}\right],
\end{aligned}
$$

and, in general,

$$
\phi^{m}(x)=\sum_{\alpha} C_{\alpha}\left\{\left(s^{\left(p_{1}\right)}\right)^{\pi_{1}}\left(s^{\left(p_{2}\right)}\right)^{\pi_{2}} \cdots\left(s^{\left(p_{\nu}\right)}\right)^{\pi_{\nu}}\right\}^{2} t^{\left(\rho_{\alpha}\right)},\left[p_{1^{\pi_{1}}} p_{2^{\pi_{2}}} \cdots p_{\nu}^{\pi_{\nu}}\right],
$$

where the notation is explained as follows:

1. $p_{i}{ }^{\pi_{i}}$ indicates that $p_{i}$ appears $\pi_{i}$ times in the partition of $m$.

2. The summation is to include all possible partitions of $m$.

3. For each term, $\sum \pi_{i}=\rho_{\alpha}$.

$$
\text { 4. } C_{\alpha}=\frac{m !}{\left(p_{1} !\right)^{\pi_{1}}\left(p_{2} !\right)^{\pi_{2}} \cdots\left(p_{\nu} !\right)^{\pi_{\nu}} \pi_{1} ! \pi_{2} ! \cdots \pi_{\nu} !} \text {. }
$$

The work of Dwyer, ${ }^{*}$ in a recent paper, proved to be of considerable assistance in obtaining a compact expression for the general derivative, as given above. It should be noted also that the numerical coefficients of the partitions which occur in our derivatives are given

* P. S. Dwyer, Moments of any rational integral isobaric sample moment function, Annals of Mathematical Statistics, vol. 8, pp. 21-65. 
in his Table II.* For example, for the fourth derivative we might have used his "weight $=4$ " and read the proper numerical coefficients from the first column, namely, $1,4,3,6,1$.

To evaluate these derivatives of $\phi(x)$ at $x=1$ it is sufficient to notice that, if the sum of the bracketed numbers is $j$, the permanent, represented by the bracket, has the value $(n-j)$ ! for $x=1$. For example, each of the bracketed quantities in $\phi^{\text {iv }}(x)$ becomes $(n-4)$ ! when $x$ is set equal to 1 .

The rest of the work necessary to calculate the moments about the mean is quite direct, although tedious. We obtain the factorial moments by dividing $\phi^{i}(x)$ by $n$ !; then, by means of well known relations, we obtain the moments about the mean. Representing the moments about the mean by $\mu_{i}$ and replacing $t$ by $n / s$, we obtain the mean and higher moments as follows:

$$
\begin{aligned}
& \bar{r}=s, \\
& \mu_{2}=\frac{s(n-s)}{n-1}, \\
& \mu_{3}= \frac{s(n-s)(n-2 s)}{(n-1)(n-2)}, \\
& \mu_{4}=\frac{s(n-s)}{(n-1)(n-2)(n-3)}\{(n-2 s)(n-3 s)(3 s+1) \\
&\left.\quad+(s-1)\left(12 n s-n-18 s^{2}-6 s\right)\right\} .
\end{aligned}
$$

Comparisons with the articles cited above indicate complete agreement between the results given for various special cases and the values obtained by the use of the formulas derived in this paper.

In conclusion, it should be mentioned that this general method can be applied to the more comprehensive case where the numbers in the $t$ sets of the matching deck are not necessarily the same but may differ from set to set. Curiously, the mean is still $s$, but the variance is decreased.

\section{Carnegie Institute of Technology}

\footnotetext{
* Loc. cit., pp. 30-32.
} 